\title{
Computer simulation study of novel chiral liquid crystal phases
}

\author{
Tanay Paul* and Jayashree Saha ${ }^{\dagger}$ \\ Department of Physics, University of Calcutta, 92, APC Road, Kolkata 700009, India
}

(Received 1 November 2018; revised manuscript received 29 July 2019; published 1 November 2019)

\begin{abstract}
We report a computer simulation study of the phase transitional behavior of a system of chiral Gay-Berne molecules embedded with a terminal dipole. In this Rapid Communication, coarse-grained modeling is done to study the influence of coupled chiral and dipolar interactions on the macroscopic liquid crystalline phase behavior. This NVT molecular dynamics simulation study presents the formation of the smectic blue phase $\left(\mathrm{BP}_{\mathrm{Sm}}\right)$, which is a comparatively recent experimentally discovered chiral phase. Our study reveals that a gradual increment of the strength of the chiral interaction brings conventional cholesteric and blue phases, whereas the addition of a dipolar interaction can induce the smectic blue phase. Moreover, we demonstrate that a further increase of dipolar strength makes novel chiral bilayer phases feasible, yet to be realized experimentally.
\end{abstract}

DOI: 10.1103/PhysRevResearch.1.032012

Chirality, a property of reflection asymmetry, pervades many branches of science, ranging from elementary particle physics to biological sciences. Molecular homochirality, i.e., the presence of only single handedness, is absolutely essential for any living system [1]. Experimental studies revealed the existence of several phases generated by chiral molecules. Important phases are the cholesteric phase $\left(N^{*}\right)$, blue phases (BP I, BP II, BP III), helical smectic $A^{*}$ phase $\left(\mathrm{Sm} A^{*}\right)$, tilted smectic $C^{*}$ phase $\left(\mathrm{Sm} C^{*}\right)$, and twisted-grain-boundary phases (TGBs) [2]. Blue phases are thermodynamically different from each other and first-order phase transitions occur between these phases [3-6].

Goodby et al. [7] suggested that the TGB model, a phase predicted by Renn and Lubensky [8], is suitable for $\operatorname{Sm} A^{*}$, where the grain boundaries of periodic arrangements of screw dislocations separate different blocks consisted of $\mathrm{Sm} A$ layers. An experimentally discovered [9] mesophase of thermotropic chiral liquid crystal is the smectic blue phase $\left(\mathrm{BP}_{\mathrm{Sm}}\right)$, which is the result of two competing frustrated mesophases. One of them is the twist-grain-boundary (TGB) phase [7,8], which is a consequence of the balance between the helical twist and the smectic order, and the other is the classical blue phase [10], where frustration occurs at the molecular level due to the impossibility of fitting molecular chirality in three dimensions.

Though chiral liquid crystal phases are well observed and characterized experimentally, a theoretical understanding at the molecular scale and its influence on phase behavior are still less understood [11]. There are a few theoretical analyses based on the molecular-statistical approach [12-17]. However, computer simulation studies based on proper modeling

\footnotetext{
*tanaypaul9492@gmail.com

†jsphy@caluniv.ac.in
}

Published by the American Physical Society under the terms of the Creative Commons Attribution 4.0 International license. Further distribution of this work must maintain attribution to the author(s) and the published article's title, journal citation, and DOI. of the chiral interaction [18-20] are necessary to assess the basic interactions responsible for the generation of these phases and to understand the relationship between their microscopic and macroscopic properties. A simulation study of the cholesteric phase incorporating the lattice model considering all rotational degrees of freedom was reported by Saha et al. [21]. Memmer et al. presented Monte Carlo simulation results of the chiral liquid crystal phases including the blue phase [22,23] by considering the coarse-grained model representing the chiral Gay-Berne fluid with an additive chiral interaction term. A recent numerical simulation study of a system of hard helical particles revealed the existence of a novel chiral phase, termed the screwlike phase [24]. Many important chiral molecules including cholesterol and other cholesteryl compounds, showing liquid crystalline behavior, have a permanent dipole in addition to chirality [25]. To the best of our knowledge, the combined effect of chiral, dipolar, and dispersive interactions on liquid crystal phases has yet to be studied theoretically. In this Rapid Communication, we have performed molecular dynamics simulations for a system of molecules interacting through the coarse-grained model interactions to understand the joint effect of chiral, dipolar, and dispersive interactions, at the molecular scale, on the liquid crystal phase properties including the smectic blue phase and the novel chiral bilayer phase, a very important phase for studying biomembranes.

In our coarse-grained molecular dynamics simulation study, we have considered molecules of an ellipsoidal shape with a chiral center at the center of mass and having a single terminal point dipole. The orientation of the dipole is perpendicular to the molecular long axis. The total interaction between the two molecules $i$ and $j$, the centers of mass of which are separated by $\vec{r}_{i j}$ and long axes unit vectors $\hat{u}_{i}$ and $\hat{u}_{j}$, is represented by the pair potential,

$$
\begin{aligned}
U\left(\vec{r}_{i j}, \hat{u}_{i}, \hat{u}_{j}\right)= & -c 4 \epsilon\left(\hat{r}_{i j}, \hat{u}_{i}, \hat{u}_{j}\right) \rho_{i j}^{-7}\left\{\left(\hat{u}_{i} \times \hat{u}_{j}\right) \cdot \hat{r}_{i j}\right\}\left(\hat{u}_{i} \cdot \hat{u}_{j}\right) \\
& +\frac{1}{r_{d}^{3}}\left[\vec{\mu}_{d_{i}} \cdot \vec{\mu}_{d_{j}}-\frac{3}{r_{d}^{2}}\left(\vec{\mu}_{d_{i}} \cdot \vec{r}_{d}\right)\left(\vec{\mu}_{d_{j}} \cdot \vec{r}_{d}\right)\right] \\
& +4 \epsilon\left(\hat{r}_{i j}, \hat{u}_{i}, \hat{u}_{j}\right)\left(\rho_{i j}^{-12}-\rho_{i j}^{-6}\right) .
\end{aligned}
$$


Here, the first term is the chiral interaction pair potential $\left(U_{C}\right)$, a pseudoscalar $[16,22,26]$, which produces an intermolecular torque of a particular handedness depending on the sign of the chirality strength parameter $c$, the value of which is zero for achiral molecules. The second term represents the electrostatic interaction between two molecules having permanent dipoles [27-29]. Here, $\vec{r}_{d}=r_{d} \hat{r}_{d}$ is the separation vector joining the two point dipoles embedded on molecules $i$ and $j$ and the respective dipole moment vectors are $\vec{\mu}_{d_{i}} \equiv \mu^{*} \hat{u}_{d_{i}}$ and $\vec{\mu}_{d_{j}} \equiv \mu^{*} \hat{u}_{d_{j}}$, respectively, where $\mu^{*}=\left(\mu^{2} / \varepsilon_{0} \sigma_{0}^{3}\right)^{1 / 2}$ is the dimensionless dipole moment of the point dipoles. The off-center point dipoles are fixed on the long axis of each molecule at a distance $\sigma_{0}$ from the center of mass [30]. The reaction field method [31] is used to incorporate the long-range nature of the dipolar interaction. The effect of the dipoles of the molecules outside a cutoff sphere $\mathscr{R}$ of radius $r_{\mathrm{RF}}$ on the dipole at the center of the sphere is considered as if the molecules outside the sphere $\mathscr{R}$ form a dielectric continuum $\left(\epsilon_{\mathrm{RF}}\right)$ producing a reaction field inside the sphere. The magnitude of the reaction field acting on molecule $i$ is proportional to the moment of the cutoff sphere $\mathscr{R}$ surrounding the $i$ th molecule and is given by [32]

$$
\overrightarrow{\mathscr{E}}_{i}=\frac{2\left(\epsilon_{\mathrm{RF}}-1\right)}{2 \epsilon_{\mathrm{RF}}+1} \frac{1}{r_{\mathrm{RF}}^{3}} \sum_{j \in \mathscr{R}} \vec{\mu}_{j}
$$

The contribution to the energy of the $i$ th molecule due to the reaction field is $-\frac{1}{2} \vec{\mu}_{i} \cdot \overrightarrow{\mathscr{E}}_{i}$ and the torque applied on the $i$ th molecule due to this reaction field is $\vec{\mu}_{i} \times \overrightarrow{\mathscr{E}}_{i}$. The dipolar cutoff radius and the dielectric constant of the medium outside the cutoff sphere $\mathscr{R}$ are $r_{\mathrm{RF}}=0.5$ and $\epsilon_{\mathrm{RF}}=1.5$ [28], respectively. The last term corresponds to the Gay-Berne interaction potential $\left(U_{\mathrm{GB}}\right)$ [33]. The separation and orientationdependent term in both $U_{C}$ and $U_{\mathrm{GB}}$ contains $\rho_{i j}=\left[r_{i j}-\right.$ $\left.\sigma\left(\hat{r}_{i j}, \hat{u}_{i}, \hat{u}_{j}\right)+\sigma_{0}\right] / \sigma_{0}$. The Gay-Berne parameters used here are $\kappa=3, \kappa^{\prime}=1 / 5, \mu=1$, and $v=2$.

We have used the $N V T$ molecular dynamics simulation method with scaled density $\rho^{*}\left(\rho^{*} \equiv \frac{N \sigma_{0}^{3}}{V}\right)$ which has been set to 0.30 . For a fixed chirality strength parameter $c, \mu^{*}$ has been varied to study the effect of the dipole moment strength on the phase behavior of such molecules. For each set of values of $c$ and $\mu^{*}$, the simulation run has been started from a well-equilibrated isotropic phase and then the scaled temperature ( $T^{*} \equiv k_{B} T / \varepsilon_{0}, k_{B}$ being the Boltzmann constant) has been decreased gradually in order to obtain a more ordered phase. At a particular temperature stage, to get the equilibrium configuration a run of $10^{6}$ steps has been performed, where the initial configuration is the previous higher-temperature equilibrium configuration, and the required averages have been calculated from another $10^{5}$ steps. The average energy of the system at each time step has been calculated and its variation with time steps has been checked, which fluctuates around a mean value which remains within $2 \%$ rms fluctuation at equilibrium [34].

We have used both a conventional cubic periodic boundary condition and its twisted modification to simulate the cholesteric phase and compared the results. Discrete fixed twist angles were employed in previous simulation studies of the cholesteric phase to get the twisted boundary condition [18,35-39]. Here, to get the twisted boundary condition, we have extended our lattice model approach [40] in this case of off-lattice study. For the twisted boundary condition a molecule belonging to an arbitrary plane has been given a rotation $\hat{e}^{\prime}=R(\beta d) \hat{e}$, where $R(\beta d)$ is the rotation matrix about the helical axis maintaining an appropriate sense of twist, and $\hat{e}$ and $\hat{e}^{\prime}$ are the old and new unit vectors of the molecular long axis, respectively; $\beta$ is the average twist angle per unit length along the helix axis and $d$ is the distance of that particular molecular plane from the center of the simulation box along the helical axis. Dividing the simulation box in parallel planar layers having the same thickness, perpendicular to the twist axis, the average twist angles $(\beta)$ between successive layers along the helical axis at each step of the simulation run have been calculated. Each molecule has been then rotated using the method described above by the average twist angle during minimum image calculation for that respective simulation step. The system has been equilibrated which takes $2 \times 10^{5}$ steps and after equilibration another $5 \times 10^{4}$ production steps are used to determine the phase properties.

In order to analyze the structural properties of the phases obtained, relevant rotationally invariant distribution functions have been computed. The radial distribution function or pair distribution function $g\left(r^{*}\right)=V\left\langle\sum_{i} \sum_{j \neq i} \delta\left(r^{*}-r_{i j}^{*}\right)\right\rangle / N^{2}\left(r^{*}\right.$ is the scaled intermolecular separation) [32] has been calculated. Longitudinal orientational correlation functions [41] $S_{220}\left(r_{\|}^{*} / d\right)$ and $S_{221}\left(r_{\|}^{*} / d\right)$ have been computed to find out the orientational correlations as a function of the scaled intermolecular distance $r_{\|}^{*}$ measured along an appropriately chosen reference axis and further scaled by a selected distance $d$ which is related to the periodicity of the phase studied [23].

In all simulations for a selected parameter set of $c$ and $\mu^{*}$, the initial configurations were a high-temperature isotropic phase. To check the dependence of chiral phase formation on the chirality strength parameter, we have simulated the system for different values of $c$. Phase sequences have been checked with decreasing temperature for $c=0.5$ considering a selected value of scaled dipole moment $\mu^{*}=1.0$ using twisted boundary condition. Results were checked for different system sizes: $N=500,864,1372$, and 2048 molecules. Here, by lowering the temperature, the cholesteric phase has been generated from the isotropic phase. A simulation run applying the twisted boundary condition during minimum image calculation has been started from a configuration, already obtained under a cubic periodic boundary condition [38], and then allowed to equilibrate under the twisted boundary condition. The pitch length with the twisted boundary condition is $(2.17 \pm 0.04) \times$ box length while that with the cubic periodic boundary condition is $(2.02 \pm 0.05) \times$ box length, indicating the elongation of pitch length for the twisted boundary condition. Again with a further lowering of temperature, the $\operatorname{Sm} A^{*}$ phase with positional ordering has been generated. In Fig. 1 the snapshots of the obtained phases for $N=2048$ have been presented for visualization. The cholesteric phase obtained using the twisted boundary condition is shown in Fig. 1(a) and the $\operatorname{Sm} A^{*}$ phase in Fig. 1(b). The plots of $\left\langle\cos ^{2} \theta\left(r^{*}\right)\right\rangle$ in the cholesteric phase along the helical axis as well as in planes perpendicular to the helical axis have been presented in Fig. 2, which shows the presence of nematic arrangements in planes 


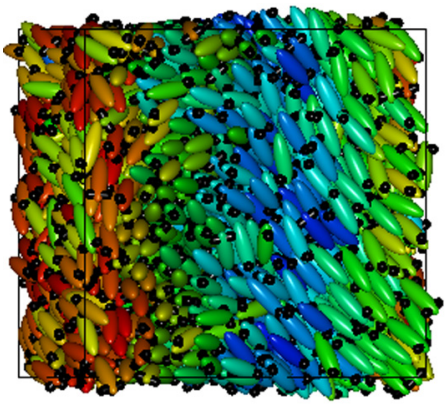

(a)

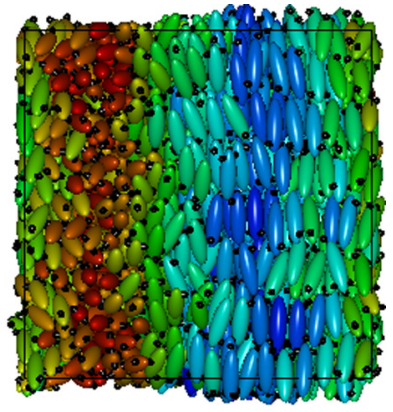

(b)

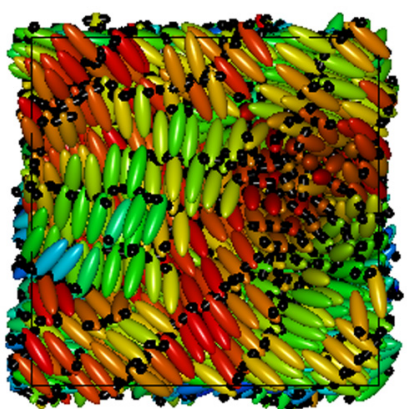

(c)

FIG. 1. Snapshots of the phases obtained for $N=2048$ molecules: For $c=0.4, \mu^{*}=1.0$ with twisted boundary condition, (a) cholesteric phase, (b) $\operatorname{Sm} A^{*}$; for $c=1.0, \mu^{*}=1.4$, (c) bilayered $\mathrm{Sm}$. Blue phase. (Molecules are presented by ellipsoids and the dipoles by short black spherocylinders.)

perpendicular to the helical axis but a relative director twist between successive planes.

At a higher value of $c=1.0$, instead of the cholesteric phase or $\mathrm{Sm} A^{*}$ phase, the smectic blue phases $\left(\mathrm{BP}_{\mathrm{Sm}}\right)$ have been generated by decreasing temperature from a blue phase. For $c=1.0$ and several values of $\mu^{*}$ from 0.1 up to 1.4 , $\mathrm{BP}_{\mathrm{Sm}}$ has been obtained. In this case, a typical phase sequence isotropic $\rightarrow \mathrm{BP} \rightarrow \mathrm{BP}_{\mathrm{Sm}}$ has been observed with decreasing temperature using the conventional cubic periodic boundary condition. A further increase of dipolar strength gives a novel bilayered phase which has been obtained for $\mu^{*}=1.4$ and $c=1.0$. Here, the bilayered smectic blue phase [Fig. 1(c)] has been found to be energetically the most favored one. For

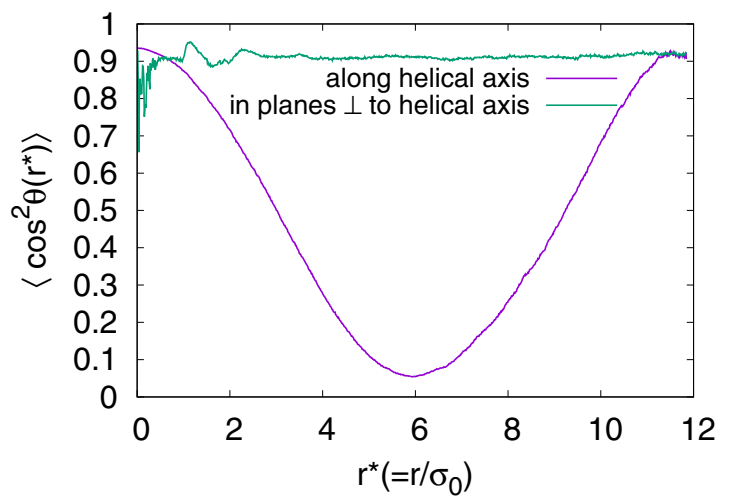

FIG. 2. Plots of $\left\langle\cos ^{2} \theta\left(r^{*}\right)\right\rangle$ in the cholesteric phase: (a) Along the helical axis, and (b) in planes perpendicular to the helical axis.

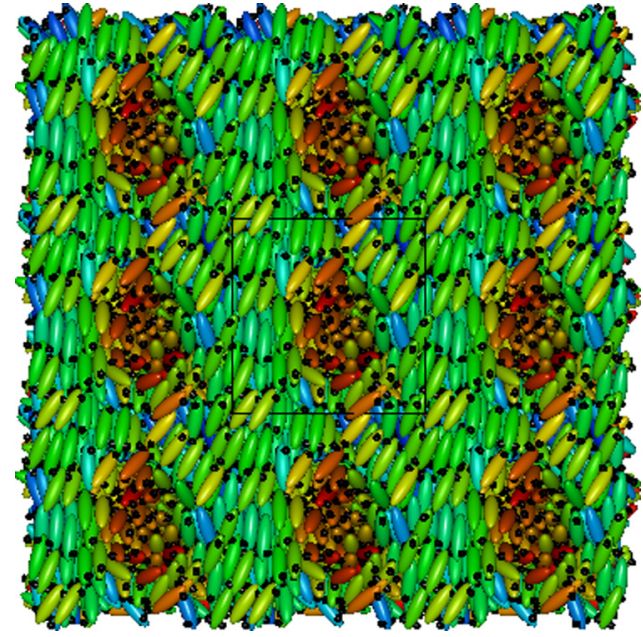

FIG. 3. Visualization of the smectic blue phase obtained with $c=1.0, \mu^{*}=1.0$ and surrounded by identical images as given by periodic boundary conditions. Molecules are shown in ellipsoids with an axis ratio of $1: 1: 3$ and dipoles by small black spherocylinders. View of one cross section perpendicular to one box face is shown.

clarity, snapshots of the smectic blue phase and bilayered smectic blue phase have been presented by periodic repetition of the simulation box $\left(\mathrm{BP}_{\mathrm{Sm}}\right.$ in Fig. 3 and bilayered $\mathrm{BP}_{\mathrm{Sm}}$ in Fig. 4), where the smectic layers are clearly visible within the blue phases. Particularly in the case of bilayered $\mathrm{BP}_{S m}$, bilayered smectic domains can be identified where dipolar ends of the molecules of two adjacent smectic layers are in opposite sides of the layers. For the system size $N=500$, the highest scaled temperatures $T^{*}$ at which the $\mathrm{BP}_{\mathrm{Sm}}$ phases occurred are given in Table I, for respective dipole strengths $\mu^{*}$. $T^{*}$ increases for higher values of $\mu^{*}$. We noted, the value of $c$ required to generate a phase is found to be lower

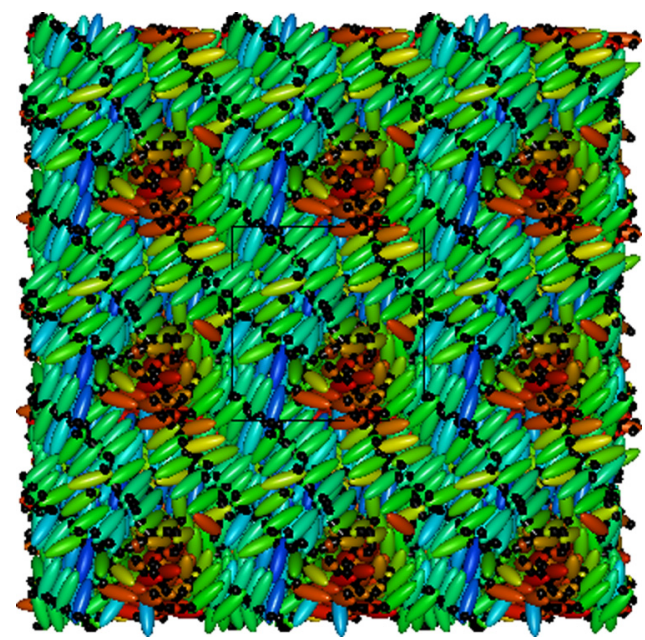

FIG. 4. Visualization of a bilayered smectic blue phase obtained with $c=1.0, \mu^{*}=1.4$ and surrounded by identical images as given by periodic boundary conditions. Molecules are shown in ellipsoids with an axis ratio of $1: 1: 3$ and dipoles by small black spherocylinders. View of one cross section perpendicular to one box face is shown. 
TABLE I. Different smectic phases formed with different sets of values of $c$ and $\mu^{*}$ at respective highest scaled temperatures $T^{*}$ at which they are stable (for $N=500$ ). The $\mathrm{Sm} A^{*}$ phase is formed by cooling it from the cholesteric phase and the smectic BPs from the blue phases by decreasing temperature.

\begin{tabular}{lcccccc}
\hline \hline$c$ & 0.5 & \multicolumn{5}{c}{1.0} \\
\hline$\mu^{*}$ & 1.0 & 0.1 & 0.5 & 0.7 & 1.0 & 1.4 \\
Phases & $\mathrm{Sm}^{*}$ & $\mathrm{BP}_{\mathrm{Sm}}$ & $\mathrm{BP}_{\mathrm{Sm}}$ & $\mathrm{BP}_{\mathrm{Sm}}$ & $\mathrm{BP}_{\text {Sm }}$ & $\mathrm{BP}_{\text {Sm }}$-bilayered \\
$T^{*}$ & 0.8 & 0.9 & 1.0 & 1.1 & 1.3 & 1.5 \\
\hline \hline
\end{tabular}

for a larger system size [23]. For example, with $N=500$ molecules the cholesteric phase has been formed with $c=0.5$ whereas with $N=2048$ it has been formed at $c=0.3$ using the twisted boundary condition. Also, a higher transition temperature has been found for larger system sizes. However, the results of various system sizes are qualitatively similar. The other physical parameters, e.g., total potential energies and pitch, are consistent in all system sizes.

For the blue phases, $S_{220}\left(r_{\|}^{*} / d\right)$ and $S_{221}\left(r_{\|}^{*} / d\right)$ computed along each of three mutually perpendicular directions of the simulation box (only one is presented in Fig. 5) show a similar behavior which indicates the phase structure of a cubic blue phase. The plot of $S_{220}\left(r_{\|}^{*} / d\right)$ (Fig. 5) starts from a positive maximum value where $r_{\|}^{*} / d=0$ and a negative minimum occurs at a separation $r_{\|}^{*}=d / 4$, where $d$ is twice the side length of the simulation box along each of the three mutually perpendicular directions of the simulation box. This indicates that the molecules separated by such a distance are more likely perpendicular to each other, referring to the existence of double twist cylinders separated by a distance of $1 / 4$ th of the lattice constant of the cubic unit cell of the blue phase along the chosen reference axis. As $d$ is equal to twice the simulation box, it can be said that the simulation box contains only one octant of the whole cubic unit cell of the blue phase.

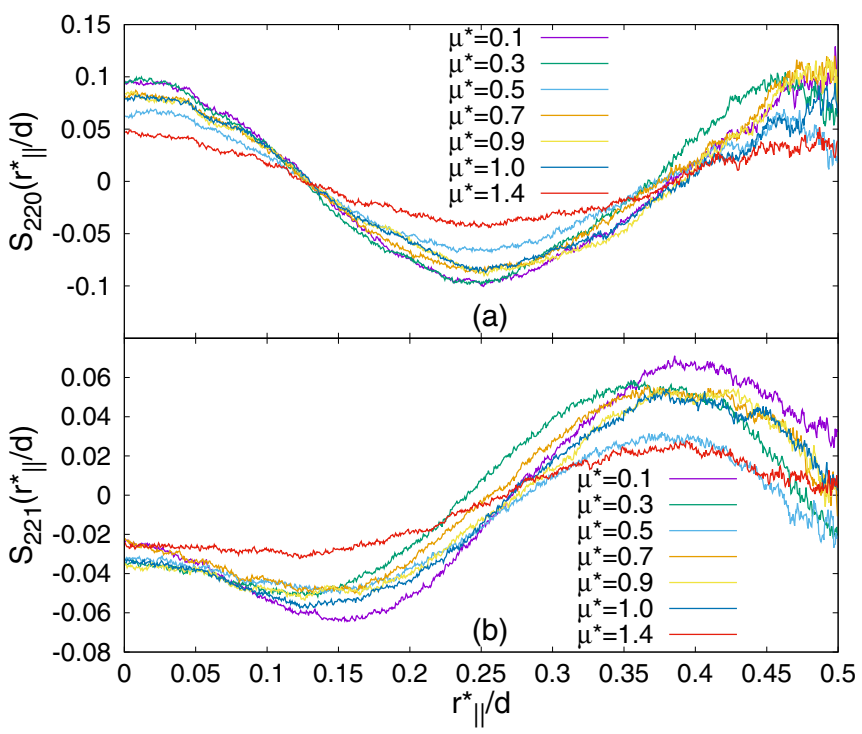

FIG. 5. Plots of (a) scalar longitudinal orientational correlation functions $S_{220}\left(r_{\|}^{*} / d\right)$, and (b) pseudoscalar longitudinal orientational correlation functions $S_{221}\left(r_{\|}^{*} / d\right)(c=1.0)$.
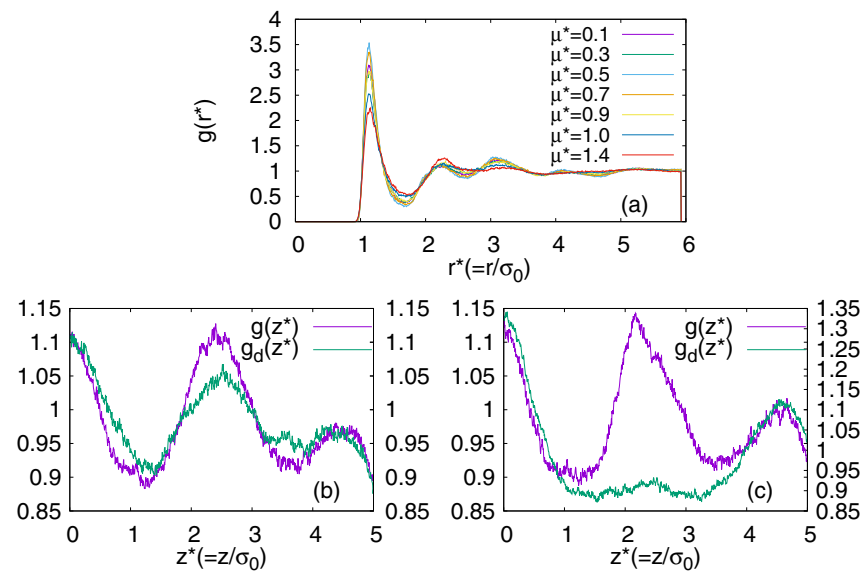

FIG. 6. Plot of (a) $g\left(r^{*}\right)$ for $c=1.0 ; g\left(z^{*}\right)$ and $g_{d}\left(z^{*}\right)$ for a spherical domain of radius $5 \sigma_{0}$ around a molecule for (b) smectic BP, peaks of both functions occurring near $z^{*}=0,2,4.5$; (c) bilayered smectic BP, peaks of $g\left(z^{*}\right)$ occurring near $z^{*}=0,2,4.5$ but those of $g_{d}\left(z^{*}\right)$ with comparable heights occurring near $z^{*}=0,4.5$.

Moreover, the occurrence of another positive maximum at a distance equal to $r_{\|}^{*}=d / 2$ refers to the presence of two parallelly oriented double twist cylinders at a separation of half the lattice constant of a cubic unit cell of the blue phase along the chosen reference axis, i.e., molecules separated by such a distance are more likely oriented parallelly. Additionally, in all the blue phases obtained, the plot of $S_{221}\left(r_{\|}^{*} / d\right)$ along one of the three mutually perpendicular directions of the simulation box (Fig. 5) has a minimum at a distance $r_{\|}^{*}=d / 8$ approximately along the reference axis, which indicates that the radius of the double twist cylinders is $1 / 8$ th of the lattice constant of the cubic unit cell of the blue phase. The analysis infers that the phases obtained are of BP I type [23]. For all the blue phases formed, the plot of $g\left(r^{*}\right)$ [Fig. 6(a)] shows short-range positional order but no order in the long range. To characterize the smectic blue phase and the bilayered smectic blue phase, the pair correlation functions, $g\left(z^{*}\right)$ for the molecular center of masses and $g_{d}\left(z^{*}\right)$ for dipolar positions [30], have been calculated, in a similar fashion as $g\left(r^{*}\right)$, inside a spherical domain of radius $5 \sigma_{0}$ along the local director of that domain and plotted in Fig. 6 [here, $z^{*}$ is the length of the projection of the separation vector between two molecular center of masses in the case of $g\left(z^{*}\right)$ and two dipolar positions in the case of $g_{d}\left(z^{*}\right)$ along the local director in reduced units] [32]. For the smectic blue phase [Fig. 6(b)], the peaks of $g\left(z^{*}\right)$ and $g_{d}\left(z^{*}\right)$ are in the same place, indicating local layer formation having random flipping of the dipolar positions in the smectic layer planes. For the bilayered smectic blue phase [Fig. 6(c)], alternate peaks for $g\left(z^{*}\right)$ coincide with the peaks of comparable heights for $g_{d}\left(z^{*}\right)$ indicating the presence of bilayered domains.

In the present molecular dynamics simulation work we have shown that fine tuning of molecular chirality coupled with a dipolar interaction can produce intermolecular forces and torques of suitable amount that can give rise to various chiral phases including a novel bilayered phase. Here, a new twisted boundary condition has been introduced for the offlattice model to study the cholesteric phase. A comparison of this new boundary condition with the periodic boundary 
condition shows that implementation of the twisted boundary condition induces a larger pitch than the system treated with the cubic periodic boundary condition. The present model not only reproduced the cholesteric phase, and $\mathrm{Sm} A^{*}$ and blue phases, but also generated the latterly discovered smectic blue phase [9]. Moreover, the simulation results show that the stabilization of a novel chiral bilayered phase has been made possible by the suitable tuning of chiral and dipolar interactions, which may give new insight into future technologically important experimental realizations of this phase.
Additionally, we hope the study may help to enrich future understanding of various other liquid crystalline phases found in biological systems, especially cholesterol-rich raft formation in cellular bilayered lipid membranes which still remains a mystery.

T.P. gratefully acknowledges the support of Council of Scientific \& Industrial Research (CSIR), India, through a Senior Research Fellowship. This work is supported by the UGC-UPE scheme of the University of Calcutta.
[1] A. A. Kornyshev, D. J. Lee, S. Leikin, and A. Wynveen, Rev. Mod. Phys. 79, 943 (2007).

[2] T. N. Govindaiah, Mol. Cryst. Liq. Cryst. 626, 115 (2016).

[3] P. P. Crooker, Liq. Cryst. 5, 751 (1989).

[4] R. Dąbrowski, Liq. Cryst. 42, 783 (2015).

[5] I. Dierking, Symmetry 6, 444 (2014).

[6] J. Cohen, Term Essays for Emergent States of Matter, University of Illinois at Urbana-Champaign, Physics 498ESM, Spring 2002, http://guava.physics.uiuc.edu/ nigel/ courses/569/Essays_2002/files/cohen.pdf (unpublished).

[7] J. W. Goodby, M. A. Waugh, S. M. Stein, E. Chin, R. Pindak, and J. S. Patel, Nature (London) 337, 449 (1989).

[8] S. R. Renn and T. C. Lubensky, Phys. Rev. A 38, 2132 (1988).

[9] E. Grelet, Liq. Cryst. Today 12, 1 (2003).

[10] W. Cao, A. Muñoz, P. Palffy-Muhoray, and B. Taheri, Nat. Mater. 1, 111 (2002).

[11] T. C. Lubensky, A. B. Harris, R. D. Kamien, and G. Yan, Ferroelectrics 212, 1 (1998).

[12] J. P. Straley, Phys. Rev. A 14, 1835 (1976).

[13] G. T. Evans, Mol. Phys. 77, 969 (1992).

[14] A. B. Harris, R. D. Kamien, and T. C. Lubensky, Phys. Rev. Lett. 78, 1476 (1997).

[15] W. J. A. Goosens, Mol. Cryst. Liq. Cryst. 12, 237 (1971).

[16] B. W. Van der Meer, G. Vertogen, A. J. Dekker, and J. G. J. Ypma, J. Chem. Phys. 65, 3935 (1976), and references therein.

[17] H. Schröder, in The Molecular Physics of Liquid Crystals, edited by G. R. Luckhurst and G. W. Gray (Academic, London, 1979), Chap. 5.

[18] S. Dussi and M. Dijkstra, Nat. Commun. 7, 11175 (2016).

[19] H. H. Wensink and L. Morales-Anda, J. Chem. Phys. 143, 144907 (2015).

[20] T. Nozawa, P. E. Brumby, and K. Yasuoka, Int. J. Mol. Sci. 19, 2715 (2018).
[21] J. Saha and M. Saha, Mol. Simul. 19, 227 (1997).

[22] R. Memmer, H.-G. Kuball, and A. Schönhofer, Liq. Cryst. 15, 345 (1993).

[23] R. Memmer, Liq. Cryst. 27, 533 (2000).

[24] G. Cinacchi, A. Ferrarini, A. Giacometti, and H. B. Kolli, J. Chem. Phys. 147, 224903 (2017).

[25] C. V. S. S. V. Gopalakrishna, C. Haranadh, and C. R. K. Murty, Trans. Faraday Soc. 63, 1953 (1967).

[26] R. Berardi, H.-G. Kuball, R. Memmer, and C. Zannoni, J. Chem. Soc., Faraday Trans. 94, 1229 (1998).

[27] R. Berardi, S. Orlandi, and C. Zannoni, Chem. Phys. Lett. 261, 357 (1996).

[28] R. Berardi, S. Orlandi, and C. Zannoni, Int. J. Mod. Phys. C 10, 477 (1999).

[29] T. K. Bose and J. Saha, Phys. Rev. Lett. 110, 265701 (2013).

[30] T. Paul and J. Saha, Phys. Rev. E 95, 062703 (2017).

[31] L. Onsager, J. Am. Chem. Soc. 58, 1486 (1936).

[32] M. P. Allen and D. J. Tildesley, Computer Simulation of Liquids (Oxford University Press, Oxford, UK, 1989).

[33] J. G. Gay and B. J. Berne, J. Chem. Phys. 74, 3316 (1981).

[34] M. Yoneya and H. J. C. Berendsen, J. Phys. Soc. Jpn. 63, 1025 (1994).

[35] M. P. Allen and A. J. Masters, Mol. Phys. 79, 277 (1993).

[36] M. P. Allen, Phys. Rev. E 47, 4611 (1993).

[37] M. P. Allen, M. A. Warren, and M. R. Wilson, Phys. Rev. E 57, 5585 (1998).

[38] R. Memmer, H.-G. Kuball, and A. Schönhofer, Ber. BunsenGes. Phys. Chem. 97, 1193 (1993).

[39] A. Kuhnhold and T. Schilling, J. Chem. Phys. 145, 194904 (2016).

[40] J. Saha, B. Nandi, P. K. Mukherjee, and M. Saha, Mol. Cryst. Liq. Cryst. 250, 185 (1994).

[41] A. J. Stone, Mol. Phys. 36, 241 (1978). 